\title{
Kinetic Verification of the Stochastic Ion Heating Mechanism in Collisionless Magnetic Reconnection
}

\author{
Young Dae Yoon (i) and Paul M. Bellan (1) \\ California Institute of Technology, USA; yyoon@caltech.edu \\ Received 2019 November 6; accepted 2019 November 25; published 2019 December 17
}

\begin{abstract}
The origin of anomalous, non-classical ion heating during magnetic reconnection has been a longstanding problem. It is verified via fully kinetic analyses and particle-in-cell simulations that stochastic heating is the main ion heating mechanism in collisionless magnetic reconnection up to moderate guide fields. Strong in-plane Hall electric fields that form during reconnection render ion motions chaotic and de facto broaden the ion distribution function. The mechanism is consistent with numerous observed features of ion heating in reconnection, such as the preferential heating of ions with higher mass-to-charge ratios and the non-conservation of the ion magnetic moment.
\end{abstract}

Unified Astronomy Thesaurus concepts: Solar magnetic reconnection (1504); Planetary magnetosphere (997); Solar coronal heating (1989)

\section{Introduction}

Magnetic reconnection is a dynamic plasma phenomenon whereby magnetic fields change topology and convert their energy into particle energies (Yamada et al. 2010). Although magnetic reconnection has been the focus of many studies, the mechanisms underlying the energy conversion from the magnetic field to particles are still poorly understood. In particular, anomalous ion heating much faster than conventional collisional heating has frequently been observed in various astrophysical and laboratory reconnection events (Ono et al. 1996, 2011; Priest et al. 1998; Hsu et al. 2000; Lin et al. 2003; Emslie 2004; Stark et al. 2005; Yoo et al. 2013; Chai et al. 2016; Hare et al. 2017); resolving the fundamental cause of this heating remains a crucial objective of reconnection research and is an important key to tackling critical problems such as the coronal heating problem (Grotian 1939).

Five features of ion heating are observed in reconnection simulations, experiments, and observations: (i) correlation with in-plane Hall electric fields (Pei et al. 2001; Stark et al. 2005; Aunai et al. 2011; Yoo et al. 2013), (ii) non-conservation of the ion magnetic moment $\mu$ (Drake et al. 2009a, 2009b; Knizhnik et al. 2011), (iii) preferential heating of ions with higher massto-charge ratios $m_{i} / q_{i}$ (Reames \& $\mathrm{Ng}$ 2004; Mason 2007; Fiksel et al. 2009), (iv) temperature anisotropy $\left(T_{i \perp} \neq T_{i \|} ; \mathrm{Li}\right.$ et al. 1998; Drake et al. 2009b; Magee et al. 2011; Hietala et al. 2015), and (v) reduction of the heating amount under a finite guide field (background out-of-plane magnetic field; Drake et al. 2009a). Because anomalous ion heating occurs over a broad range of plasma parameters, the heating mechanism is expected to involve physical quantities that are intrinsic to the reconnection process. So far, numerous mechanisms have been proposed to explain ion heating; examples include pickup (Drake et al. 2009a, 2009b), turbulent interaction (Matthaeus et al. 1984; Shibata \& Tanuma 2001), interactions with fluctuating electric fields (Fiksel et al. 2009), remagnetization (Yoo et al. 2013), and shocks or viscous damping (Ono et al. 2011). However, the proposed mechanisms are still under serious debate. For example, as mentioned by Usami et al. (2017, Section IIIC), the pickup mechanism predicts an increase of the ion heating amount as the guide field increases, whereas observations in fact indicate that there is a reduction.
In this Letter, it is unambiguously shown via exact kinetic analyses and particle-in-cell simulations that stochastic heating (McChesney et al. 1987; Sanders et al. 1998; Stasiewicz et al. 2000; Bellan 2016) is the ion heating mechanism in a generic collisionless reconnection process up to moderate guide fields and exhibits all five features of anomalous ion heating. Stochastic heating is a single-particle mechanism where large electrostatic potential gradients in the direction perpendicular to the local magnetic field destabilize individual particle motion, rendering the particle trajectories chaotic (Stasiewicz et al. 2000; Bellan 2006, 2016). This chaotic motion broadens the velocity distribution function and effectively heats the species in a fast, strong manner. Stochastic heating has been proposed to be an ion heating source in the solar wind via Alfvén wave interactions (Stasiewicz et al. 2000; Vranjes \& Poedts 2009a, 2009b, 2010a, 2010b; Chandran et al. 2010, 2013; Bourouaine \& Chandran 2013) and has been observed in laboratory experiments (McChesney et al. 1987; Sanders et al. 1998; Chai et al. 2016). The criterion for stochastic ion heating is given by a single dimensionless parameter $\alpha(\boldsymbol{x}, t)$ where

$$
\alpha(\boldsymbol{x}, t)=\frac{m_{i}}{q_{i}|\boldsymbol{B}|^{2}}\left|\nabla_{\perp}^{2} \phi\right|>1 ;
$$

here $\nabla_{\perp}$ is the gradient perpendicular to the local magnetic field $\boldsymbol{B}=\boldsymbol{B}(\boldsymbol{x}, t)$, and $\phi=\phi(\boldsymbol{x}, t)$ is the local electrostatic potential. Equation (1) provides a spatio-temporal prediction for stochastic heating.

Stochastic heating involves the breakdown of the guidingcenter approximation and causes associated non-conservation of the magnetic moment $\mu$. As $\mu$ involves $v_{\perp}$, this mechanism preferentially increases $T_{\perp}$. In addition, according to Equation (1), ions with higher $m_{i} / q_{i}$ more easily satisfy the $\alpha>1$ criterion and so are expected to be more readily heated. Thus, stochastic heating is an enticing candidate for the anomalous ion heating mechanism in magnetic reconnection. A previous study (Yoon \& Bellan 2018) used a two-fluid, zero-plasma-beta analysis of the generalized Ohm's law to predict that stochastic ion heating occurs in antiparallel reconnection. However, until now a fully self-consistent kinetic verification has been lacking, and the relation of stochastic ion heating to finite guide fields, ion magnetic moments, and ion 

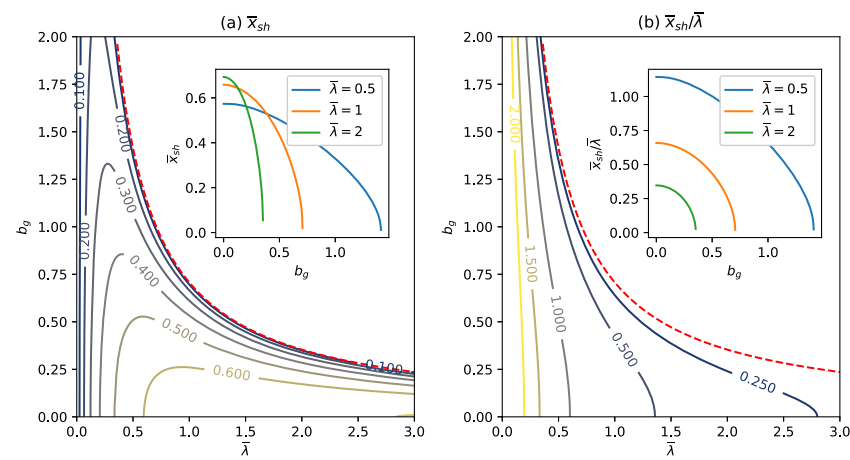

Figure 1. (a) Contours of $\bar{x}_{\mathrm{sh}}\left(\bar{\lambda}, B_{g}\right)$. Inset plots $\bar{x}_{\mathrm{sh}}$ as a function of $B_{g}$ for three $\bar{\lambda}$ values. (b) Same as (a), but for $\bar{x}_{\mathrm{sh}} / \bar{\lambda}$.

mass-to-charge ratios has not been demonstrated. This Letter fills the gap between the analytical prediction of stochastic ion heating in magnetic reconnection and a self-consistent verification by confirming the existence and inherent features of stochastic ion heating through fully kinetic analyses and simulations.

\section{Stochastic Heating in the Harris Equilibrium Current Sheet}

We begin with an analysis of the existence of stochastic heating in the Harris equilibrium current sheet (Harris 1962), which is a kinetically exact specific solution for a steady-state velocity distribution function that simultaneously satisfies Poisson's equation, Ampère's law, and the Vlasov equation. Using this specific distribution function, $\phi$ and $\boldsymbol{A}$ can be calculated, and from these the equilibrium electric and magnetic field can be derived. The equilibrium is initially solved in a frame where ions and electrons are moving at equal and opposite velocities in the $z$ direction, i.e., $V_{i z}=-V_{e z}=V$. Assuming $T=T_{i}=T_{e}$, it is seen that $\phi=0$ in this frame, and the equilibrium magnetic field is determined to have the profile (Harris 1962)

$$
B_{y}(x)=2 \sqrt{\mu_{0} n_{0} k_{B} T} \tanh \left(\frac{x}{\lambda}\right),
$$

where $\lambda=(\sqrt{2} c / V) \lambda_{D}=(\sqrt{2} c / V)\left(\lambda_{D i}^{-2}+\lambda_{D e}^{-2}\right)^{-1 / 2}=(c / V)$ $\sqrt{\epsilon_{0} k_{B} T / n_{0} e^{2}}$ is the sheath half-thickness, and $n_{0}$ is the peak density of the sheath. Lorentz transforming to a frame where $V_{i z}=0$, which is approximately the center-of-mass frame (i.e., the lab frame due to $m_{i} \gg m_{e}$ ), a lab-frame electric field with the following profile develops (Harris 1962):

$$
E_{x}(x)=-V B_{y}(x)=-2 V \sqrt{\mu_{0} n_{0} k_{B} T} \tanh \left(\frac{x}{\lambda}\right)
$$

where we have assumed $V \ll c$, so the Lorentz factor $\gamma=1$.

In this lab frame, $V_{i z}=0$ and $V_{e z}=-2 V$, so $J_{z}(x)=$ $2 n(x) e V$. Thus, Equation (3) is of the same order of magnitude as the Hall component of the electric field $E_{x, \text { Hall }}(x)=$ $-J_{z}(x) B_{y} / n(x) e=-2 V B_{y}(x)$ in the lab frame. In the vicinity of the reconnection region, the in-plane electric field is mainly balanced by the Hall component (Li \& Horiuchi 2008; Yoon \& Bellan 2018), so whether Equation (3) satisfies Equation (1) is important for determining the existence of stochastic heating.

To make the analysis more general, a guide field $B_{z}=$ $b_{g} B_{y}(x \rightarrow \infty)$ is now included as well, where $b_{g}$ is the ratio of the guide field to the asymptotic value of $B_{y}$. Because only $E_{x}(x), B_{y}(x)$, and $B_{z}$ are involved, Equation (1)

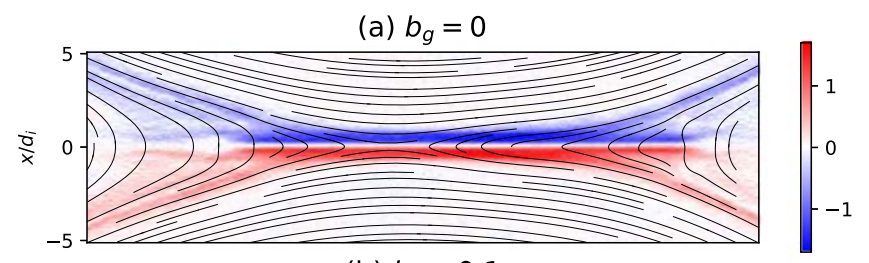

(b) $b_{g}=0.1$

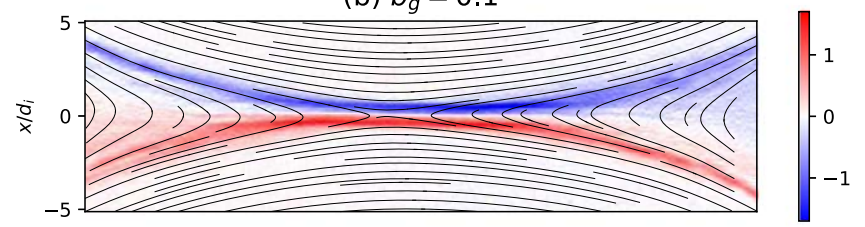

(c) $b_{g}=0.3$

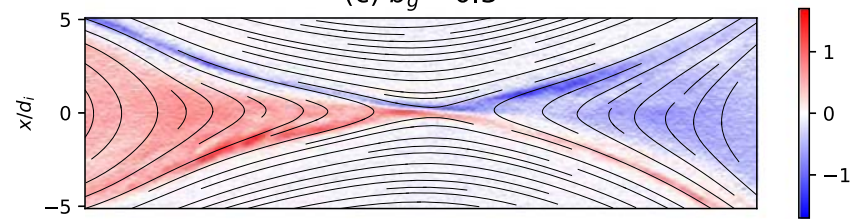

(d) $b_{g}=0.5$

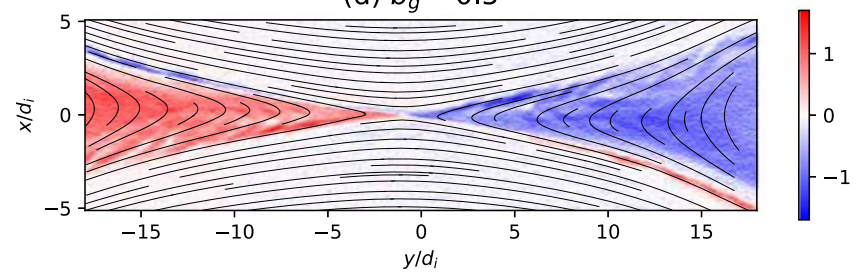

Figure 2. $\boldsymbol{B}$ (black lines) and $E_{x}$ (color) for (a) $b_{g}=0$, (b) 0.1 , (c) 0.3 , and (d) 0.5 .

becomes

$$
\frac{m_{i}}{q_{i}\left[B_{y}^{2}(x)+B_{z}^{2}\right]}\left|\frac{\partial E_{x}(x)}{\partial x}\right|>1,
$$

which, after inserting the Harris profiles (Equations (2) and (3)), becomes

$$
2 \frac{\lambda^{2}}{d_{i}^{2}} \cosh ^{2}\left(\frac{x}{\lambda}\right)\left[\tanh ^{2}\left(\frac{x}{\lambda}\right)+b_{g}^{2}\right]<1,
$$

where $d_{i}$ is the ion skin depth. Denoting $\bar{x}=x / d_{i}$ and $\bar{\lambda}=\lambda / d_{i}$, the solution to Equation (5) is $\bar{x}<\bar{x}_{\mathrm{sh}}\left(\bar{\lambda}, b_{g}\right)$ where

$$
\bar{x}_{\mathrm{sh}}=\frac{\bar{\lambda}}{2} \ln \frac{\bar{\lambda}^{2}\left(1-b_{g}^{2}\right)+1+\sqrt{\left(1-2 \bar{\lambda}^{2} b_{g}^{2}\right)\left(2 \bar{\lambda}^{2}+1\right)}}{\bar{\lambda}^{2}\left(1+b_{g}^{2}\right)} \text {. }
$$

Figure 1(a) shows contours of $\bar{x}_{\mathrm{sh}}\left(\bar{\lambda}, b_{g}\right)$, and the inset plots $\bar{x}_{\mathrm{sh}}$ as a function of $b_{g}$ for $\bar{\lambda}=0.5,1,2$. Figure 1(b) shows similar plots, but for $\bar{x}_{\mathrm{sh}} / \bar{\lambda}$, the fractional extent of the sheath that is subject to stochastic ion heating. For $b_{g}=0$ and $\bar{x} \ll \bar{\lambda}$ so that $\sinh (\bar{x} / \bar{\lambda}) \simeq \bar{x} / \bar{\lambda}$, Equation (5) simplifies to $\bar{x}<1 / \sqrt{2}$, which shows that stochastic ion heating is intrinsic to a Harris sheath. Figure 1(b) shows that the fractional heating extent increases as the sheath becomes thinner. However, Figures 1(a) and (b) show that for each $\bar{\lambda}$ there exists a maximum guide field value above which stochastic ion heating does not occur (red dashed lines). This corresponds to the argument of the square root in Equation (6) becoming negative when $b_{g}>(\sqrt{2} \bar{\lambda})^{-1}$, so no real solution for $\bar{x}_{\text {sh }}$ exists. For $\bar{\lambda}=1$, the maximum $b_{g}$ is $1 / \sqrt{2} \simeq 0.7$. 
(a) $b_{g}=0$

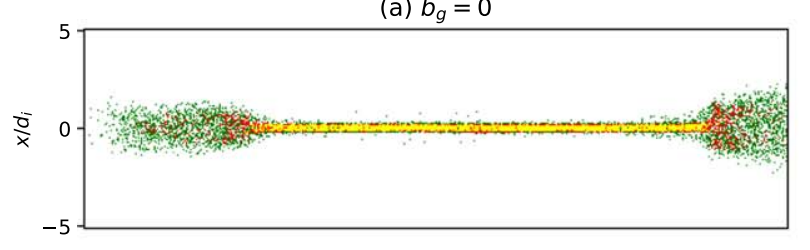

(b) $b_{g}=0.1$

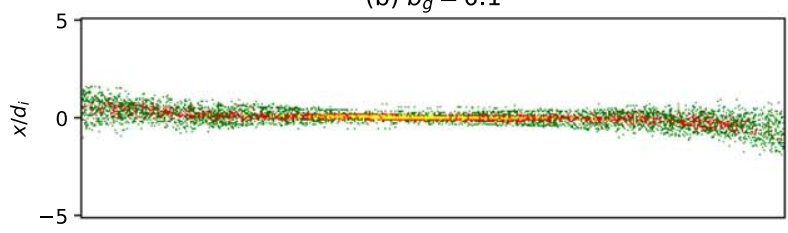

(c) $b_{g}=0.3$

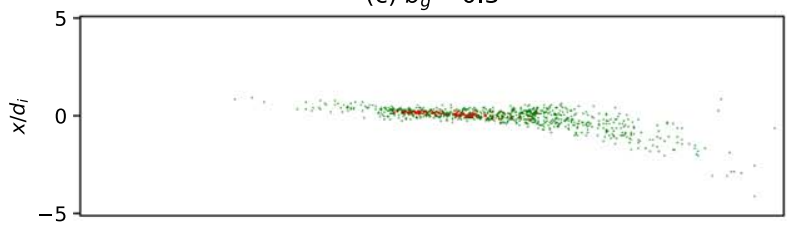

(d) $b_{g}=0.5$

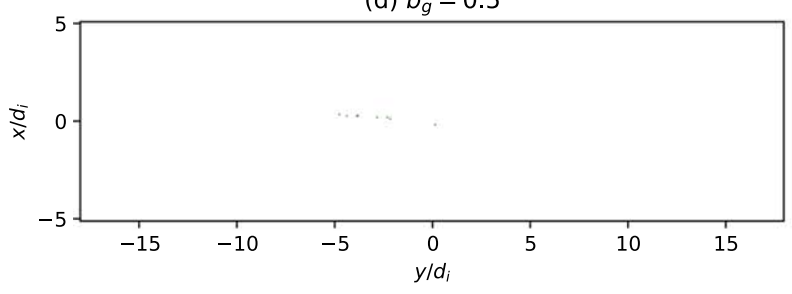

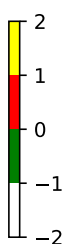
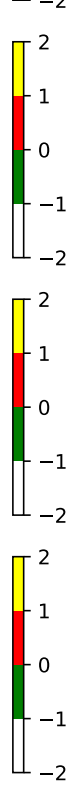

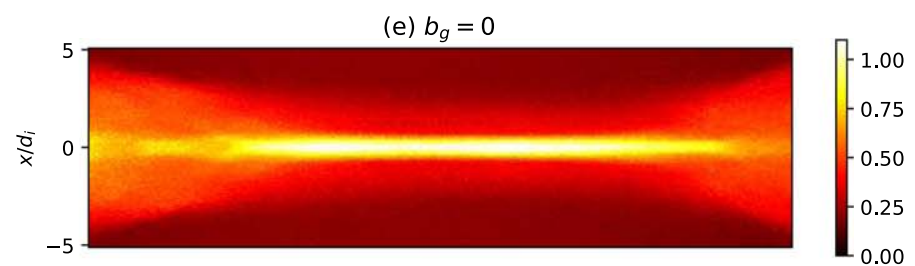

(f) $b_{g}=0.1$

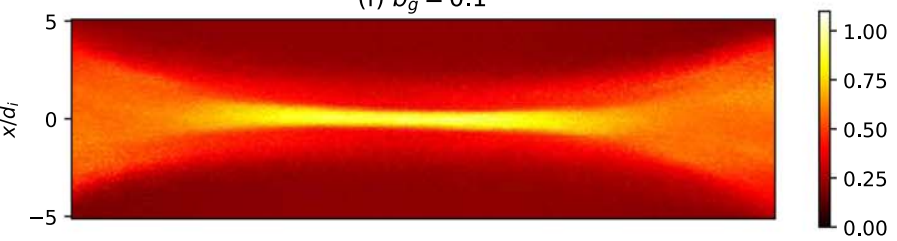

(g) $b_{g}=0.3$

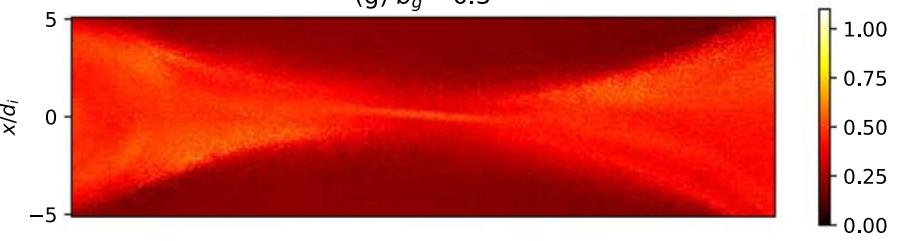

(h) $b_{g}=0.5$

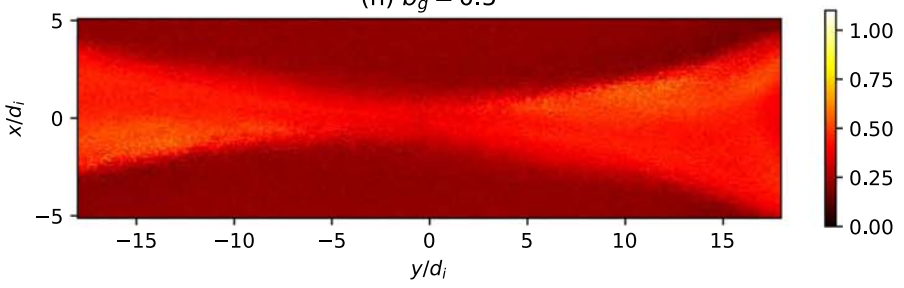

Figure 3. $\ln \alpha((\mathrm{a})-(\mathrm{d}))$ and $T_{i}((\mathrm{e})-(\mathrm{h}))$ for $b_{g}=0,0.1,0.3$, and 0.5 , respectively.

This analytical result regarding the Harris equilibrium does not directly apply to an evolving reconnection sheet because the Harris sheath does not by itself involve reconnection. However, because the Harris electric field (Equation (3)) is comparable to the Hall electric field, and the reconnecting magnetic field profile is closely related to the Harris profile (Yamada et al. 2002), one can extrapolate a general trend that the fractional extent of stochastic ion heating decreases as the guide field or the sheath width increases.

\section{Particle-in-cell Verification}

In order to test the prediction that stochastic ion heating is intrinsic to collisionless reconnection, fully kinetic particle-in-cell simulations were conducted using the SMILEI code (Derouillat et al. 2018). The simulations were conducted in 2D, i.e., $\partial / \partial z \rightarrow 0$. Lengths were normalized to $d_{i}$, time to $\omega_{c i}$, velocity to $v_{A}=d_{i} \omega_{c i}, \boldsymbol{B}$ to the upstream $B_{0}$, density to the peak density $n_{0}, T$ to $m_{i} v_{A}^{2}$, and the magnetic moment $\mu$ to $m_{i} v_{A}^{2} / B_{0}$. Double periodic Harris sheaths were employed as initial conditions, i.e., $B_{y}=B_{0}\left[\tanh (\bar{x} / \bar{\lambda})-\tanh \left(\left[\bar{x}-\bar{x}_{\max } / 2\right] / \bar{\lambda}\right)-1\right]$ and $n=n_{0}\left[\cosh ^{-2}(\bar{x} / \bar{\lambda})+\cosh ^{-2}\left(\left[\bar{x}-\bar{x}_{\max } / 2\right] / \bar{\lambda}\right)+n_{b}\right]$ where $\bar{x}_{\max }$ is the simulation box size in the $x$-direction, $n_{b}=0.2$ the relative upstream density, $\bar{\lambda}=1$ the half-thickness, and $T=T_{i}=T_{e}$ the temperature. Only one of the two sheaths is examined; the double sheath facilitates the application of periodic boundary conditions. We work in the lab frame, so an initial Harris-type electric field in the form of Equation (3) is employed. The ion-to-electron mass ratio was $m_{i} / m_{e}=100$ and the grid size was $1024 \times 2048=40.96 d_{i} \times 81.92 d_{i}$. The number of particles per cell ranged from 100 to 600 depending on the local density, and $c / v_{A}=20$. Although $\bar{x}_{\text {sh }}$ depends on both $\bar{\lambda}$ and $b_{g}$, changing $\bar{\lambda}$ requires significant alterations in simulation parameters such as the domain, and the current sheet tends to be more unstable for smaller $\bar{\lambda}$. Thus, in order to facilitate the analysis, $b_{g}$ was chosen to be the variable parameter.

Figure 2 shows $\boldsymbol{B}$ (black lines) and $E_{x}$ (color) for $b_{g}=$ (a) 0 , (b) 0.1 , (c) 0.3 , and (d) 0.5 . Times are chosen so that significant reconnection has taken place but the periodicity of the domain has not yet affected the local system. It is well known that the Hall term in the generalized Ohm's law is important in collisionless reconnection (Birn et al. 2001). Thus, strong in-plane Hall electric fields $E_{x} \sim(\boldsymbol{J} \times \boldsymbol{B})_{x}$ (color) develop; these have also been seen in previous studies (Wygant et al. 2005; Li \& Horiuchi 2008; Drake et al. 2009b; Pritchett 2010; Aunai et al. 2011; Yoon \& Bellan 2018). Although a finite guide field alters the structure, $E_{x}$ is generally pointing toward $x=0$, meaning that ions are falling down a potential valley along the inflow. A Hall $E_{y}$ (not plotted) also develops, but it is smaller in spatial extent and magnitude.

$\alpha$ as defined by Equation (1) can be calculated from the electric and magnetic field information. Figures 3(a)-(d) show $\ln \alpha$; regions where $\ln \alpha$ is above zero (red and yellow) represent the spatial extent of predicted stochastic ion heating. It is seen that the spatial extent decreases as the guide field increases, and at $b_{g}=0.5$ (Figure 3(d)), the stochasticity onset criterion is not satisfied anywhere.

Figures 3(e)-(h) show the spatial profile of the ion temperature $T_{i}=\left(2 T_{i \perp}+T_{i \|}\right) / 3$. Figures 3(e) and (f) show strong heating around the regions predicted by Figures 3(a) and (b). Figure 3(g) also shows heating, but its amount is much reduced, as predicted from there being only sparse red regions in Figure 3(c). Figure 3(h) shows that there is no strong heating at $b_{g}=0.5$, in agreement with the prediction of Figure 3(d). $T_{i \perp}$ (not plotted) exhibits similar ion heating profiles and trend. 


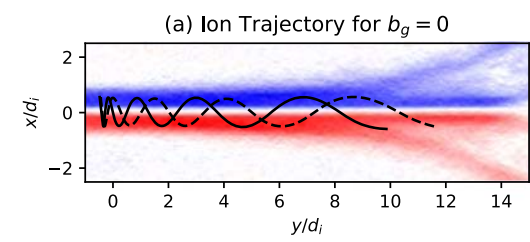

(b) Spatial Separation

(c) $\mu_{\mathbf{E} \times \mathbf{B}}$ (blue) and $\ln (\alpha)$ (red)

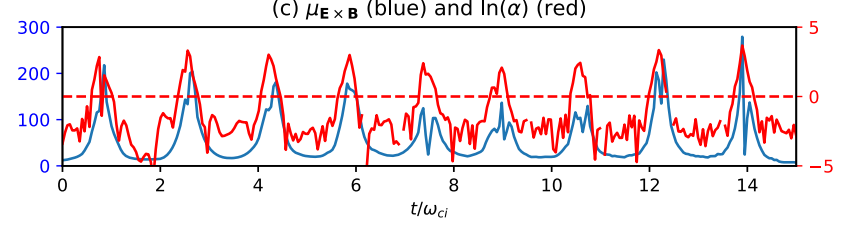

(d) Ion Trajectory for $b_{g}=0.3$

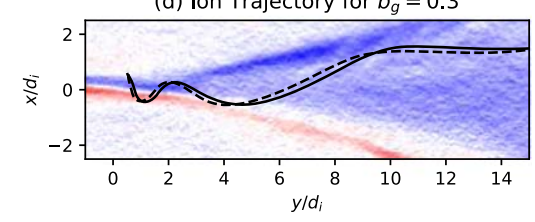

(e) Spatial Separation

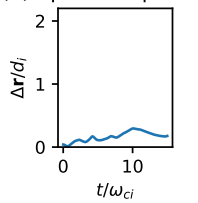

(f) $\mu_{\mathbf{E} \times \mathbf{B}}$ (blue) and $\ln (\alpha)$ (red)

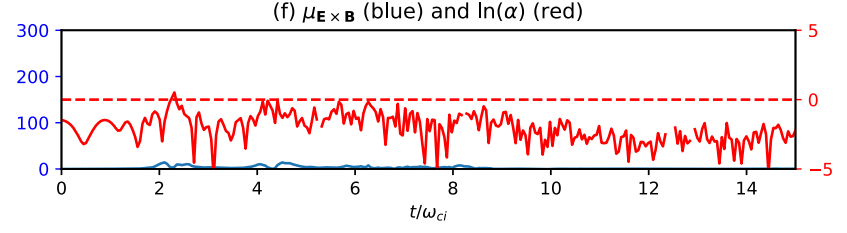

(g) Heavy Ion Trajectory for $b_{g}=0.3$

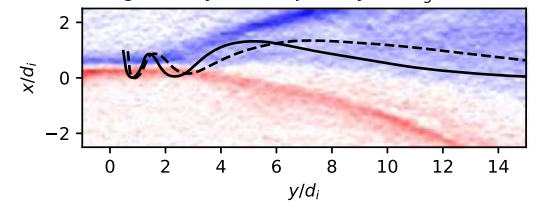

(h) Spatial Separation

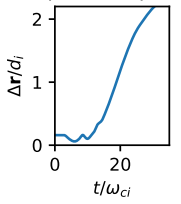

(i) $\mu_{\mathbf{E} \times \mathbf{B}}$ (blue) and $\ln (\alpha)$ (red)

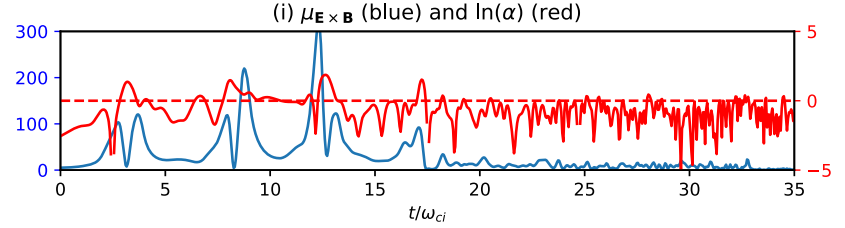

Figure 4. (a) Two test ion trajectories (black solid and dashed lines) and $E_{x}$ (color) under $b_{g}=0$. (b) The time-dependent spatial separation between the ions in (a). (c) $\mu_{\boldsymbol{E} \times \boldsymbol{B}}$ (blue) and $\ln \alpha$ (red) along the particle trajectory represented by the black solid line in (a). The red dashed line represents the stochastic heating criterion, above which stochastic heating is expected. (d)-(f) are the same as (a)-(c), respectively, except for $b_{g}=0.3$. (g)-(i) are the same as (d)-(f), except for $m_{i} / m_{e}=500$.

The correlation between perpendicular heating and the stochastic criterion involves the non-conservation of the ion magnetic moment $\mu=m_{i} v_{\mathrm{L}}^{2} / 2 B$, where $v_{\mathrm{L}}$ is the Larmor velocity that must be calculated in the frame moving with the guiding-center velocity (Bellan 2006). However, the guiding center under zero guide field is ill defined for outflowing ions because they are not magnetized. Therefore, we define a pseudo-moment $\mu_{\boldsymbol{E} \times \boldsymbol{B}}=m_{i}\left|\boldsymbol{v}_{\perp \perp}-\boldsymbol{v}_{\boldsymbol{E} \times \boldsymbol{B}}\right|^{2} / 2 B$, whose nonconservation measures the departure from guiding-center motion and thus the breakdown of the drift hierarchy. Stochastic heating involves this very breakdown (Bellan 2016), so the correlation between $\mu_{\boldsymbol{E} \times \boldsymbol{B}}$ and $\alpha$ indicates the existence of stochastic heating.

Another indication of stochastic heating is the Lyapunov exponent (Lyapunov 1992), which describes the rate of separation of two initially infinitesimally close particles. A
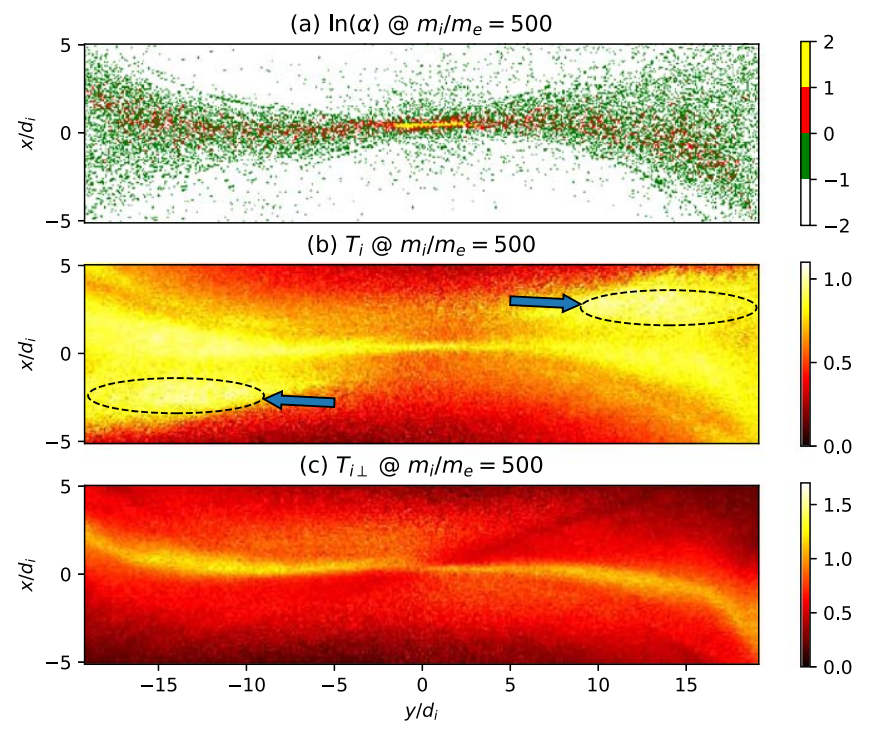

Figure 5. (a) $\ln \alpha$, (b) $T_{i}$, (c) $T_{i \perp}$ for ions with $m_{i} / m_{e}=500$ under $b_{g}=0.3$.

positive Lyapunov exponent is characteristic of chaotic behavior. In fact, Stasiewicz et al. (2000) showed that if Equation (1) is satisfied, the Lyapunov exponent is real and positive.

\section{Confirmation of Stochastic Motion}

To verify stochastic heating at a single-particle level, testparticle simulations were conducted. Figure 4(a) shows two typical ion outflow trajectories (black solid and dashed line) with an initial spatial separation of $0.04 d_{i}$ for $b_{g}=0$. Both ions undergo oscillatory motion in the $x$-direction under the Hall electric field (color; Wygant et al. 2005; Drake et al. 2009b; Aunai et al. 2011; Yoo et al. 2013; Yoon \& Bellan 2018). Figure 4(b) shows the time-dependent spatial ion separation, which exhibits divergence and thus a positive Lyapunov exponent. Figure 4(c) shows $\mu_{\boldsymbol{E} \times \boldsymbol{B}}$ (blue) and $\ln \alpha$ (red) along the particle trajectory represented by the black solid line in Figure 4(a). The red dashed line represents the stochastic heating criterion, above which $\mu_{\boldsymbol{E} \times \boldsymbol{B}}$ violation is expected. The locations where the stochastic criterion is satisfied coincides with kicks in $\mu_{\boldsymbol{E} \times \boldsymbol{B}}$.

The correlation between $\alpha$ and chaotic behavior becomes even more obvious in Figures 4(d)-(f), which are respectively the same as Figures 4(a)-(c) except for $b_{g}=0.3$ (recall from Figure $3(\mathrm{~g})$ that there is limited stochastic heating in this case). In Figure 4(e), it is apparent that the spatial separation of the ions does not diverge, illustrating non-stochastic behavior. In Figure 4(f), $\ln \alpha$ rarely goes above zero, and $\mu_{\boldsymbol{E} \times \boldsymbol{B}}$ is relatively conserved.

Finally, Equation (1) predicts that ions with larger $m_{i} / q_{i}$ more easily satisfy the stochastic heating criterion. A simulation containing a mix of heavy ions $\left(m_{i} / m_{e}=500\right)$ of density $0.1 n$ and light ions $\left(m_{i} / m_{e}=100\right)$ of density $0.9 n$ for $b_{g}=0.3$ was run. Figure 5(a) shows $\ln \alpha$ for the heavy ions, which, because of their larger $m_{i} / q_{i}$, satisfy the stochastic heating criterion across a broader range compared to the light ions in Figure 3(c). Figure 5(b) shows $T_{i}$ for the heavy ions, which, in comparison to the light ions in Figure $3(\mathrm{~g})$, undergo stronger stochastic heating. The spatial profile of $T_{i}$ agrees with Figure 5(a), but there is additional heating (blue arrows) that is not due to the stochastic mechanism and which will be 
subject to future investigations. Figure 5(c) shows that $T_{i \perp}$ agrees well with the prediction from Figure 5(a), hinting that the additional heating comes from $T_{i \|}$.

Single-particle trajectories of the heavy ions confirm that stochastic heating is occurring. Figure $4(\mathrm{~g})$ is the same as Figure 4(d), but for the heavy ions. The separation divergence (Figure $4(\mathrm{~h})$ ) and the kicks in $\mu_{\boldsymbol{E} \times \boldsymbol{B}}$ in correlation with $\alpha$ (Figure 4(i)) verify chaotic behavior. At $t / \omega_{c i}>20, \ln \alpha$ is positive only sporadically, so the kicks are smaller, whereas at $t / \omega_{c i}<20, \ln \alpha$ stays positive for longer times, so the kicks are bigger.

\section{Conclusion}

The study presented here provides strong confirmation that stochastic heating is the fast ion heating mechanism in collisionless magnetic reconnection up to moderate guide fields. The heating is consistent with calculations based on the properties of the in-plane Hall electric fields that intrinsically develop during reconnection. Ion temperature profiles were found to agree with the predictions. The stochastic nature of ions was confirmed through the examination of Lyapunov exponents and the correlation between $\mu_{\boldsymbol{E} \times \boldsymbol{B}}$ violation and the stochastic heating criterion. Heavier ions were found to be heated more strongly because they more easily satisfy the criterion.

This material is based upon work supported by the NSF Solar Terrestrial Research Program via award grant 1914599 and by the Air Force Office of Scientific Research Space Science Program via award grant FA9550-17-1-0023. The computations presented here were conducted on the Caltech High Performance Cluster partially supported by a grant from the Gordon and Betty Moore Foundation.

\section{ORCID iDs}

Young Dae Yoon (iD https://orcid.org/0000-0001-8394-2076 Paul M. Bellan (iD https://orcid.org/0000-0002-0886-8782

\section{References}

Aunai, N., Belmont, G., \& Smets, R. 2011, JGRA, 116, A09232

Bellan, P. M. 2006, Fundamentals of Plasma Physics (Cambridge: Cambridge Univ. Press)
Bellan, P. M. 2016, JPIPh, 82, 615820101

Birn, J., Drake, J. F., Shay, M. A., et al. 2001, JGRA, 106, 3715

Bourouaine, S., \& Chandran, B. D. G. 2013, ApJ, 774, 96

Chai, K.-B., Zhai, X., \& Bellan, P. M. 2016, PhPl, 23, 032122

Chandran, B. D., Li, B., Rogers, B. N., Quataert, E., \& Germaschewski, K. 2010, ApJ, 720, 503

Chandran, B. D. G., Verscharen, D., Quataert, E., et al. 2013, ApJ, 776, 45

Derouillat, J., Beck, A., Pérez, F., et al. 2018, CoPhC, 222, 351

Drake, J. F., Cassak, P. A., Shay, M. A., Swisdak, M., \& Quataert, E. 2009a, ApJL, 700, L16

Drake, J. F., Swisdak, M., Phan, T. D., et al. 2009b, JGRA, 114, A05111

Emslie, A. G. 2004, JGR, 109, A10104

Fiksel, G., Almagri, A. F., Chapman, B. E., et al. 2009, PhRvL, 103, 145002

Grotian, W. 1939, NW, 27, 214

Hare, J. D., Suttle, L., Lebedev, S. V., et al. 2017, PhRvL, 118, 085001

Harris, E. G. 1962, NCim, 23, 115

Hietala, H., Drake, J. F., Phan, T. D., Eastwood, J. P., \& McFadden, J. P. 2015, GeoRL, 42, 7239

Hsu, S. C., Fiksel, G., Carter, T. A., et al. 2000, PhRvL, 84, 3859

Knizhnik, K., Swisdak, M., \& Drake, J. F. 2011, ApJL, 743, L35

Li, B., \& Horiuchi, R. 2008, PhRvL, 101, 215001

Li, X., Habbal, S. R., Kohl, J. L., \& Noci, G. 1998, ApJL, 501, L133

Lin, R. P., Krucker, S., Hurford, G. J., et al. 2003, ApJL, 595, L69

Lyapunov, A. M. 1992, IJC, 55, 531

Magee, R. M., Den Hartog, D. J., Kumar, S. T. A., et al. 2011, PhRvL, 107 065005

Mason, G. M. 2007, SSRv, 130, 231

Matthaeus, W. H., Ambrosiano, J. J., \& Goldstein, M. L. 1984, PhRvL, 53, 1449

McChesney, J. M., Stern, R. A., \& Bellan, P. M. 1987, PhRvL, 59, 1436

Ono, Y., Tanabe, H., Hayashi, Y., et al. 2011, PhRvL, 107, 185001

Ono, Y., Yamada, M., Akao, T., Tajima, T., \& Matsumoto, R. 1996, PhRvL, 76,3328

Pei, W., Horiuchi, R., \& Sato, T. 2001, PhRvL, 87, 235003

Priest, E. R., Foley, C. R., Heyvaerts, J., et al. 1998, Natur, 393, 545

Pritchett, P. L. 2010, JGRA, 115, A10208

Reames, D. V., \& Ng, C. K. 2004, ApJ, 610, 510

Sanders, S. J., Bellan, P. M., \& Stern, R. A. 1998, PhPl, 5, 716

Shibata, K., \& Tanuma, S. 2001, EP\&S, 53, 473

Stark, A., Fox, W., Egedal, J., Grulke, O., \& Klinger, T. 2005, PhRvL, 95, 235005

Stasiewicz, K., Lundin, R., \& Marklund, G. 2000, PhyS, T84, 60

Usami, S., Horiuchi, R., \& Ohtani, H. 2017, PhPl, 24, 092101

Vranjes, J., \& Poedts, S. 2009a, PhPl, 16, 092902

Vranjes, J., \& Poedts, S. 2009b, MNRAS, 400, 2147

Vranjes, J., \& Poedts, S. 2010a, ApJ, 719, 1335

Vranjes, J., \& Poedts, S. 2010b, MNRAS, 408, 1835

Wygant, J. R., Cattell, C. A., Lysak, R., et al. 2005, JGRA, 110, A09206

Yamada, M., Ji, H., Hsu, S., et al. 2002, PhPl, 7, 1781

Yamada, M., Kulsrud, R., \& Ji, H. 2010, RvMP, 82, 603

Yoo, J., Yamada, M., Ji, H., \& Myers, C. E. 2013, PhRvL, 110, 215007

Yoon, Y. D., \& Bellan, P. M. 2018, ApJL, 868, L31 